\title{
Acute Effects of Foam Rolling Exercises on Arterial Stiffness, Flexibility and Autonomic Nervous System Function in Young and Middle-Aged Women
}

\author{
Cheonok Lee ${ }^{1}$ MS, Sewon Lee ${ }^{2,3,4} \mathrm{PhD}$ \\ 'Department of Human Movement Science, Graduate School, Incheon National University, Incheon; Division of Sport Science, College of Arts \& Physical Education, \\ Incheon National University, Incheon; ${ }^{3}$ Sport Science Institute, College of Arts \& Physical Education, Incheon National University, Incheon; ${ }^{4}$ Health Promotion Center, \\ College of Arts \& Physical Education, Incheon National University, Incheon, Korea
}

PURPOSE: Arterial stiffness is an independent risk factor for cardiovascular disease and inversely associated with flexibility. Self-myofascial release (SMR) using a foam roller is a popular intervention to improve flexibility, restore the muscles, fascia, tendons, and ligaments, and regain soft-tissue extensibility. This study aimed to investigate whether a single bout of SMR has a beneficial effect on arterial stiffness and the function of the autonomic nervous system and the relationships among these variables in young and middle-aged women by age.

METHODS: Ten healthy young (23.20 \pm 0.59 years) and 10 middle-aged women $(44.50 \pm 0.91$ years) completed an instructed SMR using a foam roller. Brachial-ankle pulse wave velocity (baPWV), augmentation index (AIx), trunk flexibility, shoulder flexibility, heart rate variability, including standard deviation of normal R-R intervals (SDNN), and the root mean square of the standard deviation of the time between heartbeats (RMSSD) were measured before and after one 30-minutes bout of SMR.

RESULTS: After one bout of SMR, trunk and shoulder flexibility increased in both groups of women, while AIx decreased significantly (from $6.9 \pm 2.9$ to $-1.9 \pm 4.6 \%, p=.0072$, young women group; from $22.1 \pm 3.4$ to $14.7 \pm 1.7 \%, p=.0049$, middle-aged women group). However, the baPWV, RMSSD, and SDNN did not change. In the analysis of correlations between all measured variables, changes in AIx showed negative correlations with flexibility and SDNN.

CONCLUSIONS: This study suggests that one bout of SMR using a foam roller positively affected arterial stiffness in women by reducing AIx and improving flexibility.

Key words: Foam roller, Self-myofascial release, Arterial stiffness, Flexibility, Autonomic Nervous system

\section{INTRODUCTION}

Arterial stiffness is known as an independent risk factor for cardiovascular diseases and mortality [1]. Since the aging process induces changes in arterial wall tissues, the phenomenon of arterial wall hardening appears even in healthy individuals without any cardiovascular disease (CVD) as they get older [2]. The arterial stiffness in healthy women without any cardiovascular risk factor increases linearly with age from 20 to 70 years of age [3].
Flexibility is not only one of the components of physical fitness but also cardiorespiratory fitness and muscular strength. Recent studies indicate that flexibility is associated with arterial stiffening [4]. Flexibility is known to be affected by connective tissues such as tendons, muscles, ligaments, fasciae, and joint [5]. According to a study based on the results of 6,000 flexibility tests, the loss of trunk flexibility due to aging accelerates at the ages of 40-50 years [6]. Structurally, flexibility and arterial stiffness are determined by components such as elastin and collagen that constitute the connective tissues [4]. Increased aortic pulse wave velocity

Corresponding author: Sewon Lee Tel +82-32-835-8572 Fax+82-32-835-0788 E-mail leesewवinu.ac.kr

Received 14 Aug 2021 Revised 4 Nov 2021 Accepted 5 Nov 2021

(๓) This is an Open Access article distributed under the terms of the Creative Commons Attribution Non-Commercial License (https://creativecommons.org/licenses/by-nc/4.0// which permits unrestricted non-commercial use. distribution, and reproduction in any medium, provided the original work is properly cited. 
(PWV) is associated with structural changes in arterial walls, including connective tissues [7]. Age-related changes in muscles and arteries tend to be similar to age-related changes in the entire body [4]. A recent study demonstrated a negative correlation between trunk flexibility and arterial stiffness in middle-aged and elderly groups [8]. In addition, study findings indicating that low upper body flexibility and trunk flexibility are related to the onset of atherosclerosis have been reported [9]. Therefore, changes in arterial stiffness according to age may appear together with changes in flexibility according to age in the same individuals [8].

Aging accompanies the declines of the overall activities of women's autonomic nervous system (ANS) functions [10]. In addition, low heart rate variability (HRV) has been presented as an indicator of pathological conditions such as coronary artery diseases, congestive heart failure, aging, and diabetic neuropathy [3]. HRV is the most closely correlated with aging and decreases linearly over 90 years (10-99 years) in healthy subjects [11].

Self-myofascial release (SMR) is a therapeutic technique for the subject, not a physical therapist, to apply pressure to his/her own fasciae and muscles [12]. The most common tool used for SMR is the foam roller. SMR using a foam roller is widely used to treat myofascial restrictions and to restore soft-tissue extensibility of muscles, tendons, ligaments, and soft tissues [13]. SMR not only stretches muscles and scar tissue, but also provides benefits similar to those of stretching and massage [12]. SMR is well known to increase flexibility, either acutely or chronically $[14,15]$. According to a previous study conducted with healthy adults, one bout SMR using a foam roller improves vascular endothelial functions and arterial functions [16]. In a recent study conducted by Ketelhut et al. performing one bout SMR had beneficial effects on peripheral and central blood pressures in healthy young adults, and a study conducted by Arroyo-Morales et al. presented that performing SMR (as an active recovery) after intermittent high-intensity exercise helped the recovery of the balance of the ANS in healthy individuals [17,18].

Middle-aged women are known to have higher arterial stiffness, lower flexibility and ANS functions compared to young women [4]. However, there have been few previous studies that investigated the effects of SMR using a foam roller on the arterial stiffness, flexibility, and ANS functions of young and middle-aged women and the differences in results according to age. Therefore, we investigated the effects of one bout SMR using a foam roller on the arterial stiffness, flexibility, and ANS functions of young and middle-aged women. In addition, we examined the differences in SMR effects between the two groups according to age.

\section{METHODS}

\section{Participants}

In this study, twenty females aged 20-49 years volunteered to participate. All participants were recruited from the community around Incheon National University (INU), and they were sedentary and nonsmokers. Each participant was assigned to either a young women group $(n=10,23.20 \pm 0.59$ years) or middle-aged women group $(n=10,44.50 \pm$ 0.91 years) according to their age. We excluded participants with a previous history of diagnosis or medication for clinical CVD including ischemic heart disease and hypertension. Moreover, none of them had applied any form of SMR or associated exercise for years. All participants were informed about the entire experimental procedures and provided written informed consent. This study was approved by the Ethical Committee of INU and preceded in accordance with the guidelines for human experimentation published by the INU Institutional Review Board (permission number: 7007971-201810-003). Basic clinical characteristics of the participants are presented in Table 1 .

\section{Study procedure}

All participants visited the laboratory between 9:00 AM and 12:00 AM. All variables were measured in the following order: body composition, trunk flexibility, shoulder flexibility, blood pressure in both arms at rest, augmentation index (AIx), brachial-ankle pulse wave velocity (baPWV), Standard deviation of normal R-R intervals (SDNN) and root mean square of the standard deviation of the time between heartbeats (RMSSD) before and after 30 minutes of SMR with a form roller. All participants were instructed not to consume caffeine and to avoid intense physical activity for at least 24 hours. Measurements for all partici-

Table 1. Basic characteristics of study participants

\begin{tabular}{lccc}
\hline Variables & $\begin{array}{c}\text { Young women } \\
(\mathrm{n}=10)\end{array}$ & $\begin{array}{c}\text { Middle-aged } \\
\text { women }(\mathrm{n}=10)\end{array}$ & p value \\
\hline Age $(\mathrm{yr})$ & $23.2 \pm 0.6$ & $44.5 \pm 0.9$ & $<.0001$ \\
Height $(\mathrm{cm})$ & $163.8 \pm 2.0$ & $161.9 \pm 1.1$ & .39 \\
Weight $(\mathrm{kg})$ & $67.6 \pm 3.7$ & $61.5 \pm 1.9$ & .15 \\
BMl $\left(\mathrm{kg} / \mathrm{m}^{2}\right)$ & $25.2 \pm 1.3$ & $23.5 \pm 0.8$ & .28 \\
Body fat $(\%)$ & $33.5 \pm 2.8$ & $33.2 \pm 0.6$ & .91 \\
SBP $(\mathrm{mmHg})$ & $107.0 \pm 3.8$ & $111.2 \pm 12.3$ & .42 \\
DBP $(\mathrm{mmHg})$ & $57.6 \pm 4.0$ & $62.8 \pm 9.5$ & .18 \\
PP $(\mathrm{mmHg})$ & $49.4 \pm 6.4$ & $48.4 \pm 8.2$ & .92 \\
\hline
\end{tabular}

Values are Means \pm SEM.

BMI, body mass index; DBP, diastolic blood pressure; SBP, systolic blood pressure; PP, pulse pressure. 
pants were conducted at the same time and place in an environment with a controlled temperature of $20-23^{\circ} \mathrm{C}$ and $40-60 \%$ humidity.

\section{1) Self-myofascial release}

The participants were instructed through a SMR procedure utilizing a commercially available foam roller $\left(\mathrm{MMXIV}^{\circledR}\right.$, Korea; diameter: $15 \mathrm{~cm}$; length: $90 \mathrm{~cm}$ ). The protocol targeted the thoracic region, the lumbar region, the quadriceps region, the hamstring region, the gluteal region and the calf region. The participants performed SMR, which required them to roll the targeted muscle groups back and forth over the foam roller working the entire surface area. The intensity was adjusted by using the bodyweight to apply pressure to the soft tissues during the foam rolling. Each participant practiced 1 or 2 times to learn the correct foam rolling technique with a trainer. For foam rolling exercise, one set lasting 60 seconds were performed and each set was separated by 30 seconds of rest. All sessions were completed within about 30 minutes. The trainer supervised the participants to do the correct SMR technique. Exercise protocol of the acute SMR program is presented in Table 2.

\section{2) Measurement of basic characteristics of participants}

Height was measured using an extensometer (Sanwa, Korea) and pulse pressure (PP) in both arms was evaluated using an Accuniq BP850 (Jawon, Korea). PP was measured twice in a stable state and the average of values was used in the analysis. Body composition was evaluated using an Inbody 720 (Biospace, Korea) utilized the bioelectric impedance analysis (BIA) method. Body weight (kg), body mass index (BMI, $\mathrm{kg} / \mathrm{m}^{2}$ ) and body fat (\%) were measured and obtained from the BIA method.

\section{3) Measurement of Alx}

AIx was assessed using ShygmoCor Xcel system (AtCor Medical, Australia) to measure aortic stiffness in a non-invasive way. The participants lay in bed and rested for approximately 10 minutes with the cuff placed on the right upper arm. Before AIx measurement, participants'

Table 2. Exercise protocol of self-myofascial release using a foam roller

\begin{tabular}{ll}
\hline Exercise protocol & Target muscles \\
\hline & Thoracic region \\
& Lumbar region \\
1 bout for $60 \mathrm{sec}$ & Quadriceps region \\
Rest for $30 \mathrm{sec}$ & Hamstring region \\
Within $30 \mathrm{~min}$ & Gluteal region \\
& Calf region \\
\hline
\end{tabular}

diastolic and systolic blood pressures were measured, and then AIx was measured using SphygmoCor Xcel software. AIx was obtained by dividing the augmented aortic pressure by the aortic pulse pressure. The augmented aortic pressure is made when the forward pulse wave generated from the left ventricle during the systolic period overlaps with the reflected pulse wave returned from the peripheral arterial trees.

\section{4) Measurement of baPWV}

BaPWV, a validated arterial stiffness measurement, was assessed in the supine position after 10 minutes of rest according to the manufacturer's protocol using a VP-1000 plus (Omron, Japan) by bilaterally collecting the tibialis posterior (ankle) artery and the brachial blood pressure. Occlusion and cuffs were wrapped around both ankles and upper arms of the participant, and measurements were prepared by attaching electrodes to the left sternum. When the participant's resting heart rate reached a stable condition, arterial stiffness was measured twice with an automatic waveform analyzer, and the value was obtained by calculating the movement distance and pulse wave propagation time between the limb arteries based on height.

\section{5) Measurement of flexibility}

Trunk flexibility was measured by a sit-and-reach test using a flexibility measuring device (Hellmas, Gefran, Italy) before and after SMR. Participants sat on the floor, attaching it to their hip with legs held straight by a testing device. They put both hands on the device with their arms straight and bent forward slowly and reached as far forward as possible. Shoulder flexibility was measured by the back scratch test before and after SMR. The dominant arm stretched over the head and turned the internal rotation and the nondominant arm was at the back. Every stretching trial was applied to the farthest point for at least two seconds. The dominant and nondominant arms were measured in turn. The distance between the middle fingers overlapped was measured. Two trials were recorded, and the average was calculated. Results were recorded with an accuracy of $0.1 \mathrm{~cm}$.

\section{6) Measurement of autonomic nervous system function}

HRV was used to assess changes in the ANS. HRV analysis is a noninvasive, clinical tool for assessing the autonomic regulation of cardiac activity as well as various autonomous-related conditions. The ANS has sympathetic and parasympathetic components. SDNN and RMSSD were used as the outcome. Continuous R-R intervals from a three-lead 
ECG were recorded for the duration of lying down, and 5 minutes of R-R intervals were selected at 10, 90, and 170 minutes for analysis. Ectopic beats were excluded by beat frequency and complexity according to software recommendations (QHRV HW 10, Medeia, USA). We computed power spectral densities on all usable 100-s blocks and calculated three frequency-domain measures: (1) low-frequency power (LF, 0.04 to $0.15 \mathrm{~Hz}),(2)$ high-frequency power $(\mathrm{HF}, 0.15$ to $0.40 \mathrm{~Hz})$, (3) the ratio of low-frequency to high-frequency power (ratio of LF/HF). Data were log transformed for statistical analysis.

\section{Statistical analyses}

All data were expressed as mean \pm SEM. Normality tests were used to determine whether all data were normally distributed and the Levene test was used to check the homogeneity of variance. We used two-way analysis of variance (ANOVA) (time $\mathrm{x}$ intervention) with repeated measures. When significant interactions were observed, group differences were assessed by student's t-tests for paired values. In addition, Pearson's correlations were performed to determine the relationships between measurement variables. Statistical analyses were performed using the GraphPad Prism version 6.05 (La Jolla, USA). All statistical significance levels were set to $p<.05$.

\section{RESULTS}

\section{Basic clinical characteristics of participants}

Table 1 illustrates the basic characteristics of the participants in this study. The study included 10 young women aged $23.20 \pm 0.59$ years and 10 middle-aged women aged $44.50 \pm 0.91$ years. None of the variables including height, weight, BMI, \%Body fat, PP, systolic blood pressure (SBP), diastolic blood pressure (DBP) differed between the young and middleaged women groups except age. We confirmed that none of the participants had overt cardiometabolic disease.

\section{Arterial stiffness}

In the pre-intervention measurement, both central arterial stiffness measured by AIx $(22.1 \pm 3.4$ vs. $6.9 \pm 2.9 \%, p=.0023)$ and peripheral arterial stiffness measured by baPWV $(12.1 \pm 0.5$ vs. $9.6 \pm 0.3 \mathrm{~m} / \mathrm{s}, p=.0005)$ of the middle-aged women group were significantly higher than those of the young women group (Fig. 1A, B). The alteration in AIx was significantly different after an acute 30-minute SMR in both young and middle-aged women groups $(p<.01$, Fig. 1A). AIx significantly decreased after SMR both in the young women group (from $6.9 \pm 2.9$ to $-1.9 \pm 4.6 \%$, $p=.0072$ ) and in the middle-aged women group (from $22.1 \pm 3.4$ to 14.7 $\pm 1.7 \%, p=.0049$ ), whereas the change in baPWV after SMR was not significantly different in both groups of women (Fig. 1B).

\section{Flexibility}

The alteration in trunk flexibility was significantly different after SMR in both young and middle-aged women groups (Fig. 2A). Trunk flexibility significantly increased after SMR both in the young women group (from $9.11 \pm 3.23$ to $12.38 \mathrm{~cm}, p=.0060$ ) and in the middle-aged women group (from $7.72 \pm 1.88$ to $10.49 \pm 1.88 \mathrm{~cm}, p=.0002$ ). In addition, changes in right and left shoulder flexibility were significantly different after SMR in both groups of women (Fig. 2B, C). Right and left shoulder flexibility significantly increased after SMR in the young women group (right: from $2.60 \pm 1.35$ to $4.83 \pm 1.46 \mathrm{~cm}, p=.0054$, left: from $-3.80 \pm 1.95$ to $-0.98 \pm 1.81 \mathrm{~cm}, p=.0015)$ and in the middle-aged women group (right: from $1.63 \pm 1.13$ to $3.60 \pm 0.98 \mathrm{~cm}, p=.0001$, left: from $-1.08 \pm 1.66$ to $0.68 \pm$
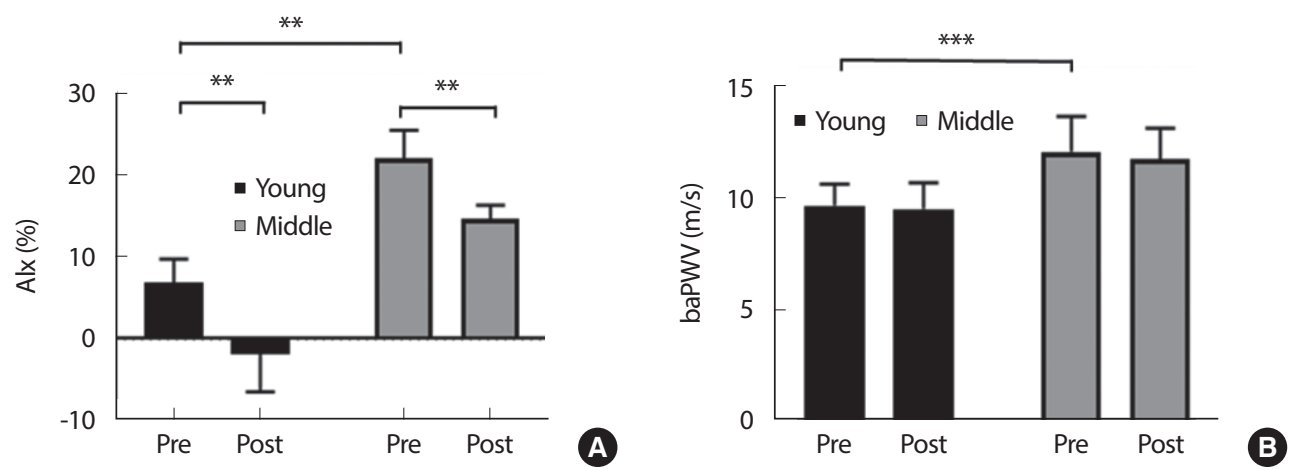

Fig. 1. Change in Alx (A) and baPWV (B) before and after an acute foam rolling in young and middle-aged women. Data are expressed as means \pm SEM. Alx, augmentation index; baPWV, brachial-ankle pulse wave velocity. Pre, before; Post, after. ${ }^{* *} p<.01,{ }^{* * *} p<.001$. 

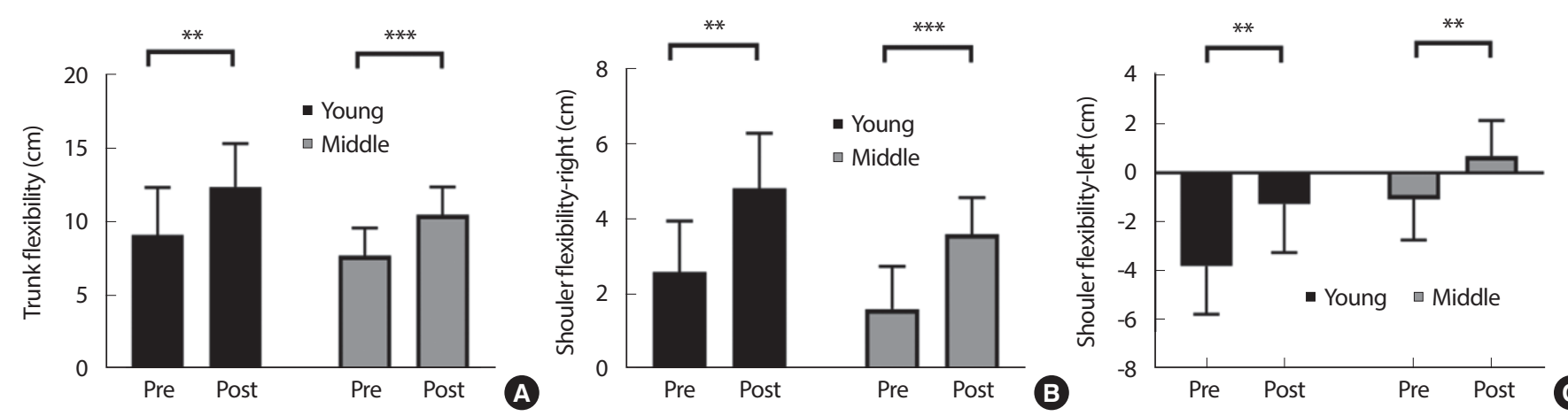

Fig. 2. Change in trunk flexibility (A), shoulder flexibility-right (B) and shoulder flexibility-left (C) before and after a foam rolling in young and middle-aged women. Data are expressed as means \pm SEM. Pre, before; Post, after. ${ }^{* *} p<.01,{ }^{* * *} p<.001$
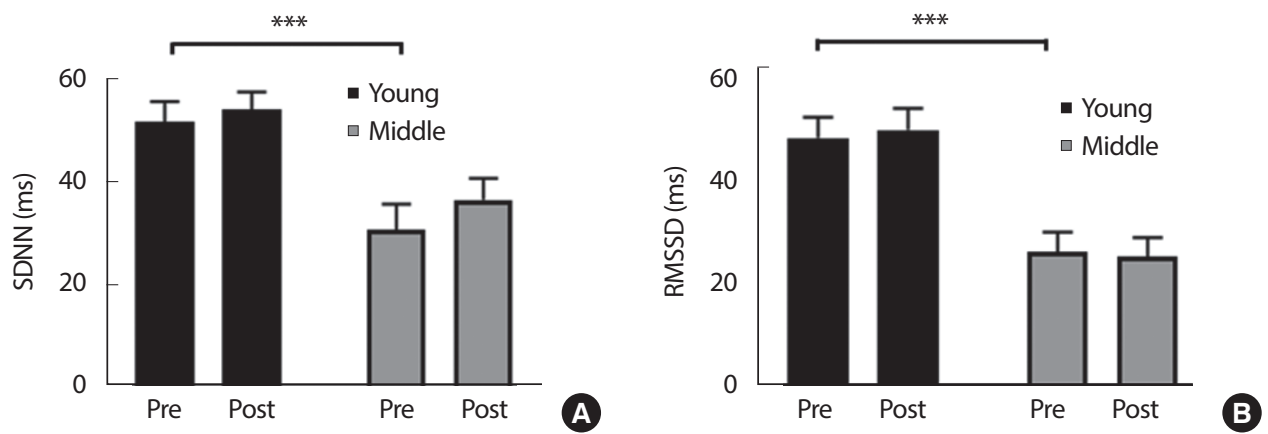

Fig. 3. Change in SDNN (A) and RMSSD (B) before and after a foam rolling in young and middle-aged women. Data are expressed as means \pm SEM. RMSSD, the root mean square of the standard deviation of the time between heart beats; SDNN, standard deviation of normal R-R intervals. Pre, before; Post, after. ${ }^{* * *} p<.001$
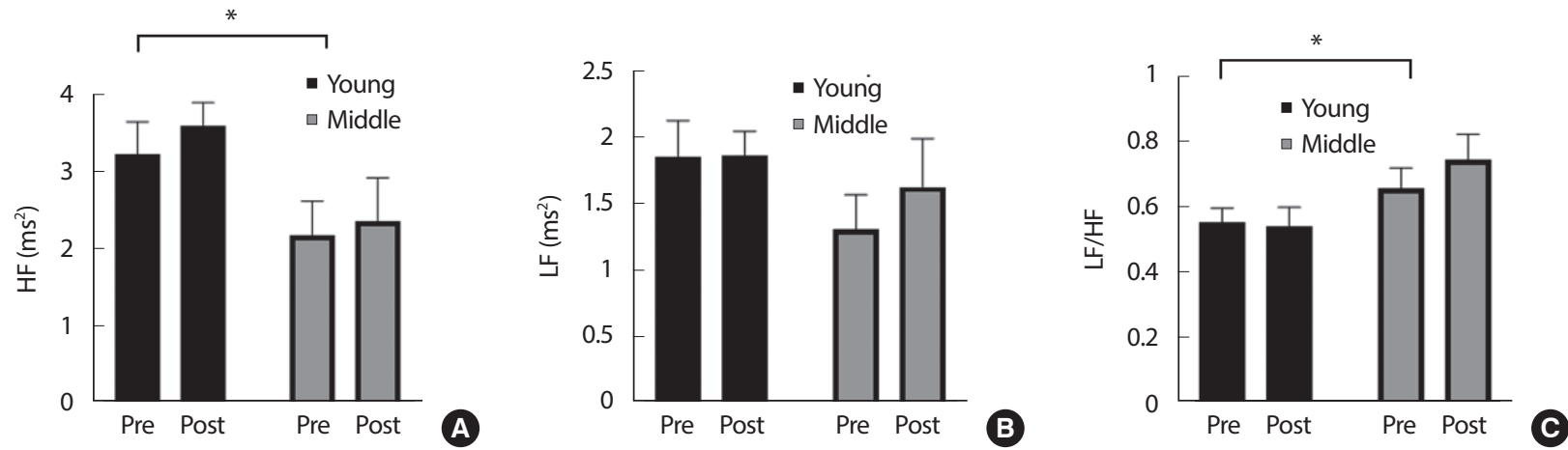

Fig. 4. Change in HF (A), LF (B) and ratio of LF/HF before and after a foam rolling in young and middle-aged women. Data are expressed as means \pm SEM. $\mathrm{HF}$, high-frequency power; LF, low-frequency power. Pre, before; Post, after. ${ }^{*} p<.05$.

$1.44 \mathrm{~cm}, p=.0012)$.

\section{Function of autonomous nervous system}

In the pre-intervention measurement, both SDNN $(27.50 \pm 3.76$ vs. $51.70 \pm 3.97 \mathrm{~ms}, p=.0003)$ and RMSSD $(23.20 \pm 3.21$ vs. $46.80 \pm 3.84 \mathrm{~ms}$, $p=.0002)$ of the middle-aged women group were significantly lower than those of the young women group. However, the alterations in both
SDNN and RMSSD after SMR were comparable in both groups of women (Fig. 3A, B). In addition, in the pre-intervention measurement, HF ( $3.22 \pm 0.44$ vs. $\left.2.17 \pm 0.48 \mathrm{~ms}^{2}, p=.0144\right)$ of the middle-aged women group were significantly lower than those of the young women group, but ratio of LF/HF $(0.55 \pm 0.05$ vs. $0.66 \pm 0.07, p=.0175)$ of the middleaged women group were significantly higher than those of the young women group (Fig. 4A, C). The alterations in LF, HF and ratio of LF/HF 
Table 3. Results of Pearson's correlation analysis

\begin{tabular}{|c|c|c|c|c|c|c|}
\hline \multirow{2}{*}{ Variables } & \multicolumn{6}{|c|}{ r-Values } \\
\hline & Alx & Trunk Flexibility & Shoulder Flexibility-right & Shoulder Flexibility-left & SDNN & RMSSD \\
\hline Alx & & $-0.491^{*}$ & $-0.585^{*}$ & -0.401 & $-0.504^{*}$ & -0.413 \\
\hline \multicolumn{7}{|l|}{ Trunk } \\
\hline Flexibility & & & $0.632^{* *}$ & -0.449 & 0.238 & 0.271 \\
\hline \multicolumn{7}{|l|}{ Shoulder } \\
\hline Flexibility-right & & & & -0.043 & 0.292 & 0.124 \\
\hline \multicolumn{7}{|l|}{ Shoulder } \\
\hline Flexibility-left & & & & & -0.086 & 0.250 \\
\hline SDNN & & & & & & $0.634^{* *}$ \\
\hline RMSSD & & & & & & \\
\hline
\end{tabular}

The correlation analysis between all measurements.

Alx, augmentation index; RMSSD, the square root of the mean square difference between N-N intervals; SDNN, standard deviation of normal R-R intervals. ${ }^{*} p<.05,{ }^{* *} p<.01$.
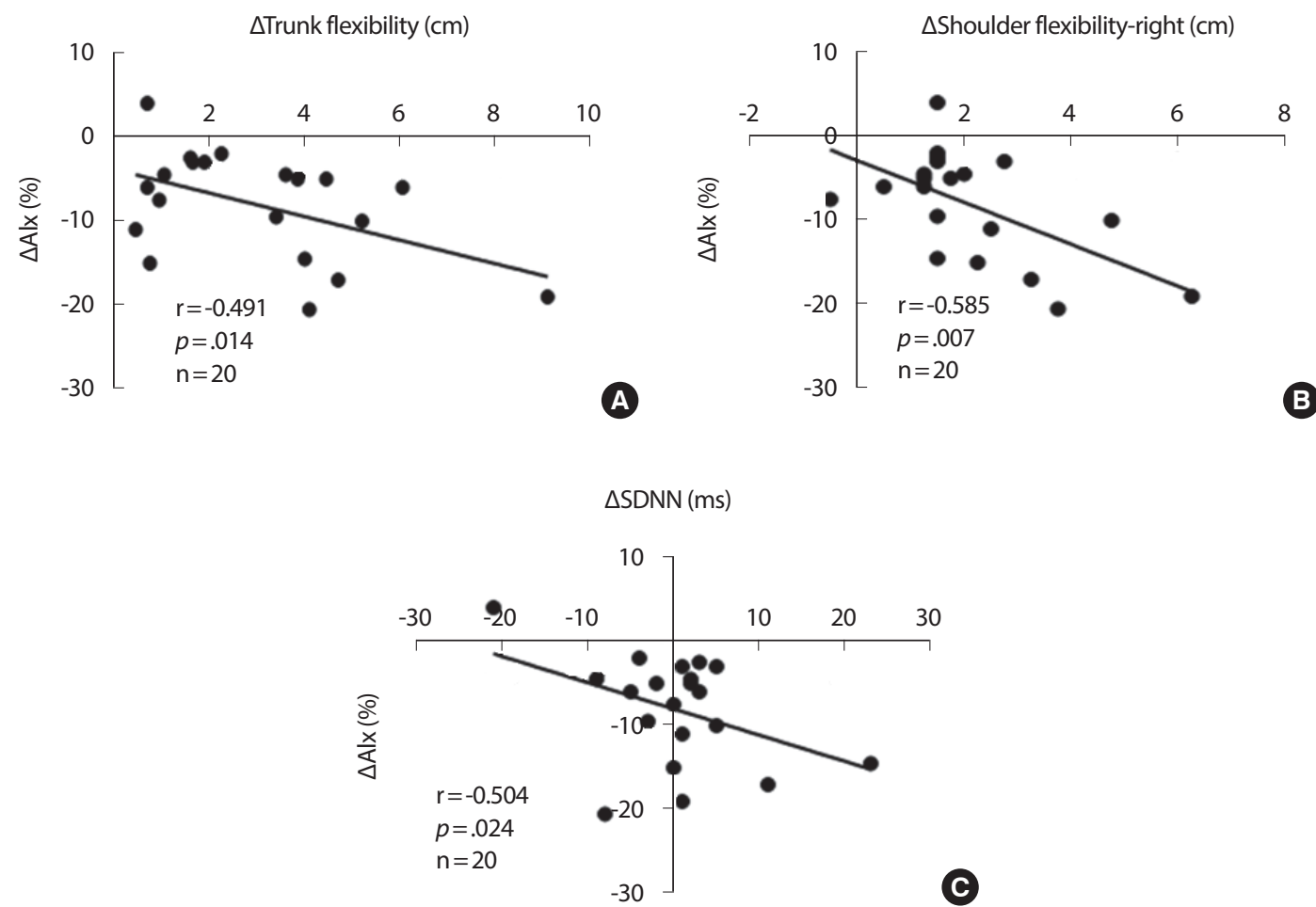

Fig. 5. Associations between Alx and trunk flexibility (A), shoulder flexibility-right (B), SDNN (C). Alx, augmentation index; SDNN, standard deviation of normal R-R intervals.

after SMR were not significantly different in both groups of women (Fig. 4).

\section{Associations}

Correlation analysis between all measurements is shown in Table 3 and Fig. 5. Specifically, AIx was negatively correlated with trunk flexibility (Fig. 5A, r=-0.491, $p=.014$ ) and more strongly with shoulder flexibility-right (Fig. 5B, r=-0.585, $p=.007$ ). Also, AIx was negatively correlated with SDNN (Fig. 5C, $r=-0.504, p=.024$ ), whereas RMSSD had no sig- nificant correlation with AIx (Table 3, $\mathrm{r}=-0.413, p=.248$ ).

\section{DISCUSSION}

Major findings of this study are as follows. In pre-intervention measurement, AIx and baPWV in the middle-aged women group were significantly higher than those in the young women group, and the SDNN, and RMSSD of the middle-aged women group were significantly lower 
than those of the young women group. After performing one bout SMR for 30 minutes, AIx significantly decreased, and flexibility increased in both groups. On the other hand, baPWV, SDNN, and RMSSD did not change in any of the group. In correlation analyses, changes in AIx showed negative correlations with flexibility and SDNN. These results suggest that one bout of SMR using a foam roller has a positive effect on arterial stiffness by reducing AIx and improving flexibility in women.

Flexibility is one of the components of physical health along with muscular strength and cardiopulmonary health [4]. Aging leads to arteriosclerosis and declines in intimal functions and plays a negative role in occurrence of CVD [19]. In a recent study, significant relationships between arterial stiffness and flexibility were observed in middle-aged and elderly people, indicating that less flexible bodies have greater arterial stiffness, especially in middle-aged and elderly people [4]. Poor upper body flexibility is associated with intima-media thickness, and poor trunk flexibility shows association with the occurrence of plaques in the common carotid artery, indicating that flexibility in middle age is associated with subclinical atherosclerosis [9]. A study conducted by Nishiwaki et al. [20] suggested a physiological mechanism indicating that both arterial stiffness and flexibility could be structurally determined by components such as elastin-collagen that constitute connective tissues and muscles. Therefore, age-related changes in arterial stiffness within the same individual may correspond to age-related changes in flexibility, and trunk flexibility is related to age-related arterial stiffness [8]. SMR using a foam roller can improve the flexibility of muscles, tendons, ligaments, and fasciae by reducing the tension of tight muscles and fasciae [21]. Recent studies focusing on the influence on flexibility have reported an increase in the range of motion for the hip joint, knee joint, and hamstring [21-23]. In this study, trunk and shoulder flexibility significantly improved after performing one bout of foam rolling in both young and middle-aged women groups. Previous studies suggested that SMR increases flexibility by increasing blood flow and soft tissue circulation $[14,21]$. During SMR, when pressure increases, mechanical receptors (i.e., Golgi tendon organs, the Ruffini and Pacinian corpuscles) stimulate the nervous system, resulting in decreases in muscle tone and increases in flexibility $[13,15]$. Additionally, in this study, right and left shoulder flexibility improved after performing one bout of SMR in both woman groups. In pre-intervention measurement, we found that there were differences in ROMs between right and left shoulder. Consistently with our findings, previous studies showed that there were significant differences in upper extremity movements and shoulder ROMs between the right and left sides [24,25]. Previous studies suggested that the overuse of some joints caused overstress in the joints and as a result, microscopic injuries that increase the deposits of scar tissues in those areas $[24,26]$. This causes a decrease in the ROM, mostly on the dominant side [26].

The present study found that the alteration of AIx, a determinant of central arterial stiffness, significantly decreased, whereas PP and baP$\mathrm{WV}$, an indicator of peripheral arterial stiffness, were not improved in both young and middle-aged women group after SMR. Okamoto et al. [16] investigated arterial stiffness and vascular endothelial function after performing one bout SMR in 10 healthy young adults. The researchers reported that baPWV which indicates arterial functions decreased, and vascular endothelial function measured with NO concentration improved [16]. In a recent study, one bout SMR in healthy young adults was shown to have beneficial effects on peripheral SBP, DBP and central SBP, augmentation pressure, and peripheral resistance [18]. In addition, acute foam rolling SMR following physical activity was shown to enhance the recovery of multiple cardiovascular variables including HRV and DBP [17]. Our study found that AIx was negatively correlated with trunk flexibility, shoulder flexibility and SDNN, respectively. Consistently with our study, previous studies reported that the improvement of flexibility was effective in reducing AIx through wave reflection magnitude, vasodilation of arteries, and vascular sympathetic activity in various populations [4,20,27,28]. Interestingly, Yamamoto et al. [8] reported that flexibility could predict arterial stiffness independently of other health components including cardiopulmonary function and muscular strength. Furthermore, repetitive stimulation of transient sympathoexcitation with habitual stretching exercises may improve arterial stiffness by chronically reducing resting sympathetic nerve activity [27,29]. In summary, SMR using a foam roller can help improve flexibility, arterial stiffness, and ANS function by relaxing the tension of muscles and fasciae. In this study, we found that AIx and baPWV in the middle-aged women group were significantly higher than those in the young women group. Consistently with our findings, previous studies showed strong correlations between age and arterial stiffness [30,31]. Central arterial stiffness also appears to begin at ages of 40-60 years and increase with age [30,31]. The reduced compliance of elastic arteries following age-related structural changes leads to decrease or failure in the aorta expansion according to the ventricular systole response [31]. In addition, SDNN and RMSSD, which indicate autonomic nervous system functions were significantly higher in the young women group than in the middle-aged women group in this study. HRV, which can be indicated by SDNN and RMS- 
SD, decreases with age [32]. HF was significantly higher in the young women group than in the middle-aged women group but ratio of LF/HF was significantly lower in the young women group than in the middleaged women group in this study. However, the alterations in LF, HF and ratio of LF/HF after SMR were not significantly different in both groups of women. HF which represents parasympathetic control and LF which represents sympathetic and parasympathetic modulation of the heart, were also shown to be higher in young women than in middle-aged women $[11,32,33]$.

Finally, the effects of SMR are found in both the young and middleaged women groups, but there was no difference in the effects of SMR by age between the groups. In a previous study, poor trunk flexibility increases arterial stiffness in young, middle, and older men, whereas the relationship is not found in young and middle-aged women [20]. Taddei et al. [34] determined endothelial function by measuring blood flow in the forearm and found an age-related decline in men but no decline in women before the menopause. In addition, lower vasoreactivity was observed at 55 years of age than at 35 years of age in women, which could be due to postmenopausal hormone changes [35].

Several important limitations in this study should be emphasized. First, because the participants were healthy young and middle-aged women, the findings may not be generalized to men or older adults. Further studies are warranted to determine the effects of SMR using a foam roller on arterial function in older adults and/or men. Moreover, the sample size of 20 participants was relatively small. More large-scale studies are needed. Second, the participants in the present study included premenopausal women. The elastic properties of central arteries are affected with the phases of the menstrual cycle [36]. However, we did not monitor menstrual phases in the present study. Thus, if premenopausal women in this population are tested during the early follicular phase, the relationship between flexibility and arterial stiffness could be analyzed more accurately. Finally, we only investigated the effects of one bout of SMR. Further investigations assessing the long-term effects of SMR are warranted and may reveal whether immediate effects can be accumulated.

\section{CONCLUSION}

In conclusion, our findings may have potentially important clinical implications. The data presented in this study suggest that one bout of SMR using a foam roller improved arterial stiffness by reducing AIx and improving flexibility in women. This study showed that AIx and baP-
$\mathrm{WV}$ of the middle-aged women were significantly higher than those of the young women. In addition, AIx had a negative correlation with trunk flexibility, shoulder flexibility and SDNN. These findings suggest that one bout of SMR using a foam roller induces short term improvements in hemodynamic parameters and has a positive effect on arterial function. We suggest that an acute SMR using a foam roller may help to reduce the risks of cardiovascular disease as a new alternative method.

\section{CONFLICT OF INTEREST}

The authors declare no conflict of interest.

\section{AUTHOR CONTRIBUTIONS}

Conceptualization: C Lee; Data curation: C Lee; Formal analysis: C Lee; Methodology: C Lee; Project administration: S Lee; Writing-original draft: C Lee; Writing-review \& editing: S Lee.

\section{ORCID}

Cheonok Lee

Sewon Lee https://orcid.org/0000-0002-0016-9514

https://orcid.org/0000-0002-6179-5156

\section{REFERENCES}

1. Sutton-Tyrrell K, Najjar SS, Boudreau RM, Venkitachalam L, Kupelian $\mathrm{V}$, et al. Elevated aortic pulse wave velocity, a marker of arterial stiffness, predicts cardiovascular events in well-functioning older adults. Circulation. 2005;111(25):3384-90.

2. Lee HY, Oh BH. Aging and arterial stiffness. Circ J. 2010:1010120923.

3. Shirai K, Hiruta N, Song M, Kurosu T, Suzuki J, et al. Cardio-ankle vascular index (CAVI) as a novel indicator of arterial stiffness: theory, evidence and perspectives. J Atheroscler Thromb. 2011;8(11):924-38.

4. Yamamoto K. Human flexibility and arterial stiffness. J Phys Fit Sports Med. 2017;6(1):1-5.

5. Alter MJ, Science of flexibility. Human Kinetics. 2004.

6. de Oliveira Medeiros HB, de Araújo DSMS, de Araújo CGS. Age-related mobility loss is joint-specific: an analysis from 6,000 Flexitest results. Age. 2013;35(6):2399-407.

7. Lakatta EG, Levy D. Arterial and cardiac aging: major shareholders in cardiovascular disease enterprises: part I: aging arteries: a "set up” for 
vascular disease. Circulation. 2003;107(1):139-46.

8. Yamamoto K, Kawano H, Gando Y, Iemitsu M, Murakami H, et al. Poor trunk flexibility is associated with arterial stiffening. Am J Physiol Heart Circ Physiol. 2009;297(4):H1314-8.

9. Suwa M, Imoto T, Kida A, Yokochi T, Iwase M, et al. Association of body flexibility and carotid atherosclerosis in japanese middle-aged men: a cross-sectional study. BMJ Open. 2018;8(1):e019370.

10. Yukishita T, Lee K, Kim S, Yumoto Y, Kobayashi A, et al. Age and sexdependent alterations in heart rate variability profiling the characteristics of men and women in their 30s. Anti-Aging Med. 2010;7(8):94-9.

11. Umetani K, Singer DH, McCraty R, Atkinson M. Twenty-four hour time domain heart rate variability and heart rate: relations to age and gender over nine decades. J Am Coll Cardiol. 1998;31(3):593-601.

12. McKenney K, Elder AS, Elder C, Hutchins A. Myofascial release as a treatment for orthopaedic conditions: a systematic review. J Athl Train. 2013;48(4):522-7.

13. Schleip R. Fascial plasticity-a new neurobiological explanation: part 1. J Bodyw Mov Ther. 2003;7(1):11-9.

14. Schleip R. Fascial plasticity-a new neurobiological explanation part 2. J Bodyw Mov Ther. 2003;7(2):104-16.

15. Lee C, Lee $\mathrm{S}$. A literature review of the effects of self-myofascial release with a foam roller on human fascial system and cardiovacular function. Exerc Sci. 2020;29(4):329-38.

16. Okamoto T, Masuhara M, Ikuta K. Acute effects of self-myofascial release using a foam roller on arterial function. J Strength Cond Res. 2014;28(1):69-73.

17. Arroyo-Morales M, Olea N, Martinez M, Moreno-Lorenzo C, DazRodrguez L, et al. Effects of myofascial release after high-intensity exercise: a randomized clinical trial. J Manipulative Physiol Ther. 2008;31 (3):217-23.

18. Ketelhut S, Möhle M, Hottenrott K. Acute effects of self-myofascial release using a foam roller on arterial stiffness in healthy young adults. Artery Res. 2020;26(4):219-22.

19. Said MA, Eppinga RN, Lipsic E, Verweij N, van der Harst P. Relationship of arterial stiffness index and pulse pressure with cardiovascular disease and mortality. J Am Heart Assoc. 2018;7(2):e007621.

20. Nishiwaki M, Kurobe K, Kiuchi A, Nakamura T, Matsumoto N. Sex differences in flexibility-arterial stiffness relationship and its application for diagnosis of arterial stiffening: a cross-sectional observational study. PLoS One. 2014;9(11):el13646.

21. MacDonald GZ, Penney MD, Mullaley ME, Cuconato AL, Drake CD, et al. An acute bout of self-myofascial release increases range of motion without a subsequent decrease in muscle activation or force. J Strength Cond Res. 2013;27(3):812-21.

22. Bushell JE, Dawson SM, Webster MM. Clinical relevance of foam rolling on hip extension angle in a functional lunge position. J Strength Cond Res. 2015;29(9):2397-403.

23. Sullivan KM, Silvey DB, Button DC, Behm DG. Roller-massager application to the hamstrings increases sit-and-reach range of motion within five to ten seconds without performance impairments. Int J Sports Phys Ther. 2013;8(3):228.

24. GÜNAL I, KÖSE N, Erdogan O, GÖKTÜRK E, Seber S. Normal range of motion of the joints of the upper extremity in male subjects, with special reference to side. JBJS. 1996;78(9):1401.

25. Macedo LG, Magee DJ. Differences in range of motion between dominant and nondominant sides of upper and lower extremities. Manipulative Physiol Ther. 2008;31(8):577-82.

26. Allander E, Björnsson O, Olafsson O, Sigfusson N, Thorsteinsson J. Normal range of joint movements in shoulder, hip, wrist and thumb with special reference to side: a comparison between two populations. Int J Epidemiol. 1974;3(3):253-61.

27. Wong A, Figueroa A. Eight weeks of stretching training reduces aortic wave reflection magnitude and blood pressure in obese postmenopausal women. J Hum Hypertens. 2014;28(4):246-50.

28. Douris PC, Ingenito T, Piccirillo B, Herbst M, Petrizzo J, et al. Martial arts training attenuates arterial stiffness in middle aged adults. Asian J Sports Med. 2013;4(3):201.

29. Yamamoto K, Kawada T, Kamiya A, Takaki H, Sugimachi M, et al. Static interaction between muscle mechanoreflex and arterial baroreflex in determining efferent sympathetic nerve activity. Am J Physiol Heart Circ Physiol. 2005;289(4):H1604-9.

30. Tanaka H, Dinenno FA, Monahan KD, Clevenger CM, DeSouza CA, et al. Aging, habitual exercise, and dynamic arterial compliance. Circulation. 2000;102(11):1270-5.

31. Monahan KD, Tanaka H, Dinenno FA, Seals DR. Central arterial compliance is associated with age-and habitual exercise-related differences in cardiovagal baroreflex sensitivity. Circulation. 2001;104(14): $1627-32$.

32. Moodithaya SS, Avadhany ST. Comparison of cardiac autonomic activity between pre and post menopausal women using heart rate variability. Indian J Physiol Pharmacol. 2009;53(3):227-34.

33. Yamazaki T, Asanoi H, Ueno H, Yamada K, Takagawa J, et al. Central 
sympathetic inhibition augments sleep-related ultradian rhythm of parasympathetic tone in patients with chronic heart failure. Circ J. 2005;69(9):1052-6.

34. Taddei S, Virdis A, Ghiadoni L, Mattei P, Sudano I, et al. Menopause is associated with endothelial dysfunction in women. Hypertension. 1996;28(4):576-82.
35. Jensen-Urstad K, Johansson J. Gender difference in age-related changes in vascular function. J Intern Med. 2001;250(1):29-36.

36. Hayashi K, Miyachi M, Seno N, Takahashi K, Yamazaki K, et al. Variations in carotid arterial compliance during the menstrual cycle in young women. Exp Physiol. 2006;91(2):465-72. 